PROCEEDINGS OF THE

AMERICAN MATHEMATICAL SOCIETY

Volume 139, Number 3, March 2011, Pages 1081-1090

S 0002-9939(2010)10557-9

Article electronically published on October 1,2010

\title{
THE HORN-LI-MERINO FORMULA FOR THE GAP AND THE SPHERICAL GAP OF UNBOUNDED OPERATORS
}

\author{
G. RAMESH
}

(Communicated by Marius Junge)

\begin{abstract}
In this article we obtain the Horn-Li-Merino formula for computing the gap as well as the spherical gap between two densely defined unbounded closed operators. As a consequence we prove that the gap and the spherical gap of an unbounded closed operator are 1 and $\sqrt{2}$ respectively. With the help of these formulae we establish a relation between the spherical gap and the gap of unbounded closed operators. We discuss some properties of the spherical gap similar to those of the gap metric.
\end{abstract}

\section{INTRODUCTION}

The aim of this article is to deduce the Horn-Li-Merino formula for the gap and the spherical gap between two unbounded closed operators. The gap and the spherical gap are two equivalent metrics on the set of all closed subspaces of a Hilbert space. These allow one to discuss the convergence of subspaces and has applications in Perturbation Theory [12] and in invariant subspaces of finite dimensional operators 7 . These concepts can be applied to define a metric on the space of bounded operators between Hilbert spaces and the class of unbounded closed operators between Hilbert spaces via the graph of an operator.

These metrics play an important role in the class of unbounded closed operators, since most of the operators that arise in physical applications are unbounded (see [12, 21] for details). By the closed graph theorem [22, page 281] an everywhere defined closed operator between Hilbert spaces is bounded. Hence unbounded closed operators are defined on proper subspaces of Hilbert spaces. Because of this fact, the sum and product of unbounded closed operators need not be defined. Hence this class of operators does not form a vector space. Thus one can think of only defining a metric on this class of operators. One of the well known and widely used metrics is the gap metric. This metric was defined by Cordes and Labrousse in 3 to study the properties of unbounded semi-Fredholm operators. A metric which is stronger than the gap metric was introduced by Kaufman in [13] to study the convergence of unbounded operators in Hilbert space. Some relations between the gap metric, the metric defined by Kaufman and the metric induced by the operator

Received by the editors October 14, 2009 and, in revised form, April 2, 2010.

2010 Mathematics Subject Classification. Primary 47A55.

Key words and phrases. Closed operator, gap metric, spherical gap, Horn-Li-Merino formula.

The author is thankful to the NBHM for financial support and ISI Bangalore for providing necessary facilities to carry out this work.

(C)2010 American Mathematical Society Reverts to public domain 28 years from publication 
norm on the space of bounded operators between Hilbert spaces are discussed in 14.

On the space of bounded operators on a Hilbert space, the topology induced by the gap metric and the one induced by the operator norm are the same. Hence the gap metric is a natural generalization of the operator norm. A relation between the operator norm and the gap between two bounded operators on a Hilbert space and applications to Perturbation Theory of operators can be found in [18.

There are two formulae available in the literature for computing the gap between operators, namely the MacIntosh formula and the Horn-Li-Merino formula. A formula for the gap and the spherical gap of an operator on finite dimensional Hilbert space was derived by Habibi in [5, 6].

A more general version of the result for the gap between two $n \times n$ matrices was proved by MacIntosh, which is now well known as the MacIntosh formula (see [17). Later this result was extended to bounded operators between Hilbert spaces by Nakamoto in [18. This formula for the case of unbounded closed operators was obtained by S. H. Kulkarni and G. Ramesh in [15.

The spherical gap formula for matrices was obtained by Horn, Li and Merino in 11, which is well known as the Horn-Li-Merino formula. This formula depends on the minimum modulus (smallest singular value) of a certain matrix which depends on the given matrices. A generalization of this result to the case of bounded operators between Hilbert spaces can be found in [19].

Some relations among the gap and the spherical gap of a bounded operator on Banach spaces were studied by Cvetković in [4].

In this article we obtain the Horn-Li-Merino formula for the gap and the spherical gap beween two unbounded closed operators. In fact our results generalizes the results of Nakamoto (see Theorems 3.1 and 3.3). We prove that the gap and the spherical gap of an unbounded closed operator are 1 and $\sqrt{2}$ respectively. Finally by establishing a relation between the gap and the spherical gap of unbounded closed operators, we prove that these two metrics are equivalent and study some properties of the spherical gap metric, which are analogous to those of the gap metric.

We hope our results will be useful in the Perturbation Theory of Linear Operators, results related to invariant subspaces of unbounded closed operators as in the case of finite dimensional operators and in solving operator equations, in particular for studying the stability of the solution of an operator equation involving unbounded operators.

In the second section we set up some notation and briefly state some preliminary results used in the article. In the third section we prove the Horn-Li-Merino formula for the gap and the spherical gap and we study some properties similar to the gap metric. Finally, we illustate our theorems with examples.

\section{NOtATION AND PRELIMINARY RESUltS}

Throughout the article we consider infinite dimensional Hilbert spaces, which will be denoted by $H, H_{1}, H_{2}$, etc. The inner product and the induced norm are denoted by $\langle$,$\rangle and \|$.$\| respectively. If A: D(A) \rightarrow H_{2}$ is a linear operator with domain $D(A) \subseteq H_{1}$, then the graph $G(A)$ of $A$ is defined by $G(A):=$ $\{(x, A x): x \in D(A)\} \subseteq H_{1} \times H_{2}$. If $G(A)$ is closed, then $A$ is called a closed operator. If $D(A)$ is dense in $H_{1}$, then $A$ is called a densely defined operator. For a 
densely defined $A: D(A)\left(\subseteq H_{1}\right) \rightarrow H_{2}$, there exists a unique adjoint $A^{*}$ of $A$ which satisfies $\langle A x, y\rangle=\left\langle x, A^{*} y\right\rangle$ for all $x \in D(A)$ and $y \in D\left(A^{*}\right)$. The set of all closed operators between $H_{1}$ and $H_{2}$ is denoted by $\mathcal{C}\left(H_{1}, H_{2}\right)$. The set of all bounded operators between $H_{1}$ and $H_{2}$ is denoted by $\mathcal{B}\left(H_{1}, H_{2}\right)$. If $H_{1}=H_{2}=H_{1}$, then $\mathcal{B}\left(H_{1}, H_{2}\right)$ and $\mathcal{C}\left(H_{1}, H_{2}\right)$ are denoted by $\mathcal{B}(H)$ and $\mathcal{C}(H)$ respectively.

If $A$ and $B$ are closed operators with the property that $D(B) \subseteq D(A)$ and $B x=A x$ for all $x \in D(B)$, then $B$ is called the restriction of $A$ and $A$ is called an extension of $B$. If $M$ is a closed subspace of a Hilbert space $H$, then $P_{M}$ is the orthogonal projection $P_{M}: H \rightarrow H$ with range $M$, and the unit sphere of a closed subspace of a Hilbert space is denoted by $S_{M}:=\{x \in M:\|x\|=1\}$. If $A \in \mathcal{C}\left(H_{1}, H_{2}\right)$ is densely defined, then we define $\widehat{A}:=\left(I+A A^{*}\right)^{-1}$ and $\check{A}:=$ $\left(I+A^{*} A\right)^{-1}$.

Here we recall some of the known facts which are useful throughout.

Definition 2.1 ([12, page 197]). Let $M$ and $N$ be closed subspaces of a Hilbert space $H$. Define

$$
\theta_{0}(M, N):= \begin{cases}\sup \left\{d(x, N): x \in S_{M}\right\} & \text { if } M \neq\{0\} \\ 0 & \text { else }\end{cases}
$$

where $d(x, L)$ is the distance from $x$ to $M$. The quantity

$$
\theta(M, N):=\max \left\{\theta_{0}(M, N), \theta_{0}(N, M)\right\}
$$

is called the gap between $M$ and $N$.

Equivalently, if $P=P_{M}, Q=P_{N}$ are orthogonal projections, then $\theta(M, N)=$ $\|P-Q\|=\max \{\|P(I-Q)\|,\|Q(I-P)\|\}$ (see [1, page 70] for details).

Definition 2.2 ([12, pages 197-198]). Let $M$ and $N$ be closed subspaces of a Hilbert space $H$. Define

$$
\tilde{\theta}_{0}(M, N):= \begin{cases}\sup \left\{d\left(x, S_{N}\right): x \in S_{M}\right\} & \text { if } M \neq\{0\}, N \neq\{0\} \\ 0 & \text { if } M=\{0\}, \\ 2 & \text { if } M \neq\{0\}, N=\{0\} .\end{cases}
$$

The quantity

$$
\tilde{\theta}(M, N):=\max \left\{\tilde{\theta}_{0}(M, N), \tilde{\theta}_{0}(N, M)\right\}
$$

is called the spherical gap between $M$ and $N$.

If $A, B \in \mathcal{C}\left(H_{1}, H_{2}\right)$, then $G(A)$ and $G(B)$ are closed subspaces of $H_{1} \times H_{2}$. The gap and the spherical gap between $A$ and $B$ are defined by $\theta(A, B):=\theta(G(A), G(B))$ and $\tilde{\theta}(A, B):=\tilde{\theta}(G(A), G(B))$ respectively. In particular $\theta(A):=\theta(A, 0)$ and $\tilde{\theta}(A):=\tilde{\theta}(A, 0)$ are called the gap and the spherical gap of $A$ respectively.

Definition 2.3 (Minimum modulus [12, page 231]). Let $A \in \mathcal{C}\left(H_{1}, H_{2}\right)$ be densely defined. Then $m(A):=\inf \{\|A x\|: x \in D(A),\|x\|=1\}$ is called the minimum modulus of $A$.

Note 2.4. If $A \in \mathcal{C}\left(H_{1}, H_{2}\right)$ is densely defined, then $m\left(A^{*} A\right)=m(A)^{2}$.

Definition 2.5 (Positive square root [23, Theorem 13.31, page 349]). Let $A \in \mathcal{C}(H)$ be a positive operator. Then there exists a unique positive operator $B$ such that $A=B^{2}$. The operator $B$ is called the positive square root of $A$ and is denoted by $B=A^{\frac{1}{2}}$. 
Definition 2.6 (Spectrum [23, page 346]). Let $A \in \mathcal{C}(H)$ be densely defined. The resolvent of $A$ is defined by

$$
\rho(A):=\left\{\lambda \in \mathbb{C}: A-\lambda I: D(A) \rightarrow H \text { is bijective and }(A-\lambda I)^{-1} \in \mathcal{B}(H)\right\},
$$

and the spectrum $\sigma(A)$ is the complement of $\rho(A)$ in $\mathbb{C}$.

Proposition 2.7. Let $A \in \mathcal{C}(H)$ be selfadjoint. Then $m(A)=\inf \{|\lambda|: \lambda \in \sigma(A)\}$.

Proof. The proof of this goes along similar lines to that of [16, Theorem 3.5].

Proposition 2.8 ([23, page 336, Theorem 13.13]). Let $A \in \mathcal{C}\left(H_{1}, H_{2}\right)$ be densely defined. Then the operator $I+A^{*} A: D\left(A^{*} A\right) \rightarrow H_{1}$ is bijective and $\check{A}$ is bounded.

The following results will be used quite often throughout the article without mentioning.

Lemma $2.9\left([3,8,9,10,20)\right.$. Let $A \in \mathcal{C}\left(H_{1}, H_{2}\right)$ be densely defined. Then:

(1) $\check{A} \in \mathcal{B}\left(H_{1}\right), \widehat{A} \in \mathcal{B}\left(H_{2}\right),\|\check{A}\| \leq 1$ and $\|\widehat{A}\| \leq 1$.

(2) $\widehat{A} A \subseteq A \check{A}, \quad\|A \check{A}\| \leq \frac{1}{2}$ and $\check{A} A^{*} \subseteq A^{*} \widehat{A}, \quad\left\|A^{*} \widehat{A}\right\| \leq \frac{1}{2}$.

(3) If $g \in C[0,1]$, then $g(\check{A}) A^{*} \subseteq A^{*} g(\widehat{A})$ and $g(\widehat{A}) A \subseteq A g(\check{A})$.

Theorem 2.10 ([15, Theorem 3.5]). Let $A, B \in \mathcal{C}\left(H_{1}, H_{2}\right)$ be densely defined. Then the operators $B \check{B}^{\frac{1}{2}} \check{A}^{\frac{1}{2}}, \widehat{B}^{\frac{1}{2}} A \check{A}^{\frac{1}{2}} A \check{A}^{\frac{1}{2}} \check{B}^{\frac{1}{2}}$ and $\widehat{A}^{\frac{1}{2}} B \check{B}^{\frac{1}{2}}$ are bounded, and

$$
\theta(A, B)=\max \left\{\left\|B \check{B}^{\frac{1}{2}} \check{A}^{\frac{1}{2}}-\widehat{B}^{\frac{1}{2}} A \check{A}^{\frac{1}{2}}\right\|,\left\|A \check{A}^{\frac{1}{2}} \check{B}^{\frac{1}{2}}-\widehat{A}^{\frac{1}{2}} B \check{B}^{\frac{1}{2}}\right\|\right\} .
$$

\section{MAin Results}

In this section we prove the main results. Recall that if $A: D(A)\left(\subseteq H_{1}\right) \rightarrow H_{2}$ is densely defined, then $\check{A}=\left(I+A^{*} A\right)^{-1}$ and $\widehat{A}=\left(I+A A^{*}\right)^{-1}$.

Theorem 3.1 (The Horn-Li-Merino formula for the spherical gap). Let $A, B \in$ $\mathcal{C}\left(H_{1}, H_{2}\right)$ be densely defined. Let $\Gamma(A, B)=\check{A}^{\frac{1}{2}} \check{B}^{\frac{1}{2}}+A^{*} \widehat{A}^{\frac{1}{2}} B \check{B}^{\frac{1}{2}}$. Then $\Gamma(A, B)$ is bounded, and

$$
\tilde{\theta}(A, B)=\sqrt{2-2 \min \{m(\Gamma(A, B)), m(\Gamma(B, A))\}} .
$$

Proof. The operators $\check{A}^{\frac{1}{2}}$ and $\widehat{B}^{\frac{1}{2}}$ are bounded, and hence $\check{A}^{\frac{1}{2}} \widehat{B}^{\frac{1}{2}}$ is bounded. By Lemma 2.9, $A^{*} \widehat{A}^{\frac{1}{2}}$ and $B \check{B}^{\frac{1}{2}}$ are bounded; hence $A^{*} \widehat{A}^{\frac{1}{2}} B \check{B}^{\frac{1}{2}}$ is bounded. Thus $\Gamma(A, B)$ is bounded.

By definition,

$$
\begin{aligned}
\tilde{\theta}_{0}(A, B) & =\sup _{(x, A x) \in S_{G(A)}} \inf _{(y, B y) \in S_{G(B)}}\|(x, A x)-(y, B y)\| \\
& =\sup _{(x, A x) \in S_{G(A)}} \inf _{(y, B y) \in S_{G(B)}}\left(\|x-y\|^{2}+\|A x-B y\|^{2}\right) .
\end{aligned}
$$

For $x \in D(A)$ and $y \in D(B)$, consider

$$
\begin{aligned}
f(x, y): & =\sqrt{\|x-y\|^{2}+\|A x-B y\|^{2}} \\
& =\sqrt{\|x\|^{2}+\|y\|^{2}-2 \operatorname{Re}\langle x, y\rangle+\|A x\|^{2}+\|B y\|^{2}-2 \operatorname{Re}\langle A x, B y\rangle} \\
& =\sqrt{\|x\|^{2}+\|A x\|^{2}+\|y\|^{2}+\|B y\|^{2}-2 \operatorname{Re}(\langle x, y\rangle+\langle A x, B y\rangle)} .
\end{aligned}
$$


Thus $\tilde{\theta}_{0}(A, B)=\sup _{\|x\|^{2}+\|A x\|^{2}=1} \inf _{\|y\|^{2}+\|B y\|^{2}=1} f(x, y)$.

Since the operators $\check{A}^{\frac{1}{2}}: H_{1} \rightarrow D(A)$ and $\check{B}^{\frac{1}{2}}: H_{1} \rightarrow D(B)$ are bijective, there exist $u, v \in H_{1}$ such that $x=\check{A}^{\frac{1}{2}} u$ and $y=\check{B}^{\frac{1}{2}} v$. It can be easily verified that $\|x\|^{2}+\|A x\|^{2}=\|u\|^{2}$ and $\|y\|^{2}+\|B y\|^{2}=\|v\|^{2}$.

So

$$
\begin{aligned}
f(x, y) & =\sqrt{\|u\|^{2}+\|v\|^{2}-2 \operatorname{Re}\left(\left\langle\check{A}^{\frac{1}{2}} u, \check{B}^{\frac{1}{2}} v\right\rangle+\left\langle A \check{A}^{\frac{1}{2}} u, B \check{B}^{\frac{1}{2}} v\right\rangle\right)} \\
& =\sqrt{\|u\|^{2}+\|v\|^{2}-2 \operatorname{Re}\left(\left\langle u, \check{A}^{\frac{1}{2}} \check{B}^{\frac{1}{2}} v\right\rangle+\left\langle u, A^{*} \widehat{A}^{\frac{1}{2}} B \check{B}^{\frac{1}{2}} v\right\rangle\right)} \\
& =\sqrt{\|u\|^{2}+\|v\|^{2}-2 \operatorname{Re}(\langle u, \Gamma(A, B) v\rangle)} \\
& =: g(u, v) .
\end{aligned}
$$

Hence $\tilde{\theta}_{0}(A, B)=\sup _{u \in H_{1},\|u\|=1} \inf _{v \in H_{1},\|v\|=1} g(u, v)$. Using the Cauchy-Schwarz inequality, we get

$$
\inf _{\|v\|=1} g(u, v)=\sqrt{1+\|u\|^{2}-2\left\|\Gamma(A, B)^{*} u\right\|} .
$$

Taking the supremum over $u$ such that $\|u\|=1$, we have

$$
\tilde{\theta}_{0}(A, B)=\sup _{\|u\|=1} \inf _{\|v\|=1} g(u, v)=\sqrt{2-2 \inf \left\|\Gamma(A, B)^{*} u\right\|}
$$

It can be easily verified that $\Gamma(A, B)^{*}=\Gamma(B, A)$. Hence

$$
\tilde{\theta}_{0}(A, B)=\sqrt{2-2 m(\Gamma(B, A))}
$$

Interchanging the roles of $A$ and $B$ we get

$$
\tilde{\theta}_{0}(B, A)=\sup _{\|v\|=1} \inf _{\|u\|=1} g(u, v)=\sqrt{2-2 m(\Gamma(A, B))} .
$$

The conclusion follows from the definition of $\tilde{\theta}(A, B)$.

Remark 3.2. Theorem 3.1 generalizes the formula given by Horn, Li and Merino for $m \times n$ matrices (see [11] for details), whereas Nakamoto [19] proved that if $A, B \in$ $\mathcal{B}(H)$, then $\tilde{\theta}(A, B)=\sqrt{2-2 \min \{m(\Gamma(A, B)), m(\Gamma(B, A))\}}$, where $\Gamma(A, B):=$ $\check{A}^{\frac{1}{2}}\left(I+A^{*} B\right) \check{B}^{\frac{1}{2}}$. By using the relations in Lemma 2.9 , it can be shown that this result and Theorem 3.1 coincide for $A, B \in \mathcal{B}(H)$.

Theorem 3.3 (The Horn-Li-Merino formula for the gap). Let $A, B \in \mathcal{C}\left(H_{1}, H_{2}\right)$ be densely defined. Then

$$
\theta(A, B)=\sqrt{1-\min \left\{m(\Gamma(A, B))^{2}, m(\Gamma(B, A))^{2}\right\}}
$$

where $\Gamma(A, B)$ is as in Theorem 3.1 . 
Proof. Let $P:=P_{G(A)}$ and $Q:=P_{G(B)}$. Then by [15, Theorem 3.5], we have $\|P(I-Q)\|=\left\|A \check{A}^{\frac{1}{2}} \check{B}^{\frac{1}{2}}-\widehat{A}^{\frac{1}{2}} B \check{B}^{\frac{1}{2}}\right\|$. So

$$
\begin{aligned}
\|P(I-Q)\|^{2}= & \left\|\left(A \check{A}^{\frac{1}{2}} \check{B}^{\frac{1}{2}}-\widehat{A}^{\frac{1}{2}} B \check{B}^{\frac{1}{2}}\right)^{*}\left(A \check{A}^{\frac{1}{2}} \check{B}^{\frac{1}{2}}-\widehat{A}^{\frac{1}{2}} B \check{B}^{\frac{1}{2}}\right)\right\| \\
= & \left\|\left(\check{B}^{\frac{1}{2}} A^{*} \widehat{A}^{\frac{1}{2}}-B^{*} \widehat{B}^{\frac{1}{2}} \widehat{A}^{\frac{1}{2}}\right)\left(A \check{A}^{\frac{1}{2}} \check{B}^{\frac{1}{2}}-\widehat{A}^{\frac{1}{2}} B \check{B}^{\frac{1}{2}}\right)\right\| \\
= & \| \check{B}^{\frac{1}{2}} A^{*} \widehat{A}^{\frac{1}{2}} A \check{A}^{\frac{1}{2}} \check{B}^{\frac{1}{2}}-\check{B}^{\frac{1}{2}} A^{*} \widehat{A} B \check{B}^{\frac{1}{2}} \\
& -B^{*} \widehat{B}^{\frac{1}{2}} \widehat{A}^{\frac{1}{2}} A \check{A}^{\frac{1}{2}} \check{B}^{\frac{1}{2}}+B^{*} \widehat{B}^{\frac{1}{2}} \widehat{A} B \check{B}^{\frac{1}{2}} \| \\
= & \| \check{B}^{\frac{1}{2}} A^{*} A \check{A} \check{B}^{\frac{1}{2}}-\check{B}^{\frac{1}{2}} A^{*} \widehat{A} B \check{B}^{\frac{1}{2}} \\
& -B^{*} \widehat{B}^{\frac{1}{2}} A \check{A} \check{B}^{\frac{1}{2}}+B^{*} \widehat{B}^{\frac{1}{2}} \widehat{A} B \check{B}^{\frac{1}{2}} \| .
\end{aligned}
$$

Let $\tilde{\Gamma}(A, B)=\Gamma(A, B) \Gamma(A, B)^{*}$. It is easy to verify that

$$
\tilde{\Gamma}(A, B)=\check{A}^{\frac{1}{2}} \check{B} \check{A}^{\frac{1}{2}}+\check{A}^{\frac{1}{2}} B^{*} \widehat{B} A \check{A}^{\frac{1}{2}}+A^{*} \widehat{A}^{\frac{1}{2}} B \check{B} \check{A}^{\frac{1}{2}}+A^{*} \widehat{A}^{\frac{1}{2}} B B^{*} \widehat{B} A \check{A}^{\frac{1}{2}} .
$$

Note that $I-\tilde{\Gamma}(B, A)=I-\check{B}^{\frac{1}{2}} \check{A} \check{B}^{\frac{1}{2}}-\check{B}^{\frac{1}{2}} A^{*} \widehat{A} B \check{B}^{\frac{1}{2}}-B^{*} \widehat{B}^{\frac{1}{2}} A \check{A} \check{B}^{\frac{1}{2}}-$ $B^{*} \widehat{B}^{\frac{1}{2}} A A^{*} \widehat{A} B \check{B}^{\frac{1}{2}}$. We claim that $\left\|(P(I-Q))^{*}(P(I-Q))\right\|=\|I-\tilde{\Gamma}(B, A)\|$. It is enough to show that $\check{B}^{\frac{1}{2}} A^{*} A \check{A} \check{B}^{\frac{1}{2}}+B^{*} \widehat{B}^{\frac{1}{2}} \widehat{A} B \check{B}^{\frac{1}{2}}=I-\check{B}^{\frac{1}{2}} \check{A}^{\frac{1}{2}}-B^{*} \widehat{B}^{\frac{1}{2}} A A^{*} \widehat{A} B \check{B}^{\frac{1}{2}}$.

Consider

$$
\begin{aligned}
& I-\check{B}^{\frac{1}{2}} A^{*} A \check{A} \check{B}^{\frac{1}{2}}-B^{*} \widehat{B}^{\frac{1}{2}} \widehat{A} B \check{B}^{\frac{1}{2}}=\check{B}^{\frac{1}{2}} \check{A} \check{B}^{\frac{1}{2}}-\check{B}^{\frac{1}{2}} \check{A} \check{B}^{\frac{1}{2}}+I-\check{B}^{\frac{1}{2}} A^{*} A \check{A} \check{B}^{\frac{1}{2}} \\
& -B^{*} \widehat{B}^{\frac{1}{2}} \widehat{A} B \check{B}^{\frac{1}{2}} \\
& =\check{B}^{\frac{1}{2}} \check{A} \check{B}^{\frac{1}{2}}+I-\check{B}^{\frac{1}{2}}\left(\check{A}+A^{*} A \check{A}\right) \check{B}^{\frac{1}{2}} \\
& -B^{*} \widehat{B}^{\frac{1}{2}} \widehat{A} B \check{B}^{\frac{1}{2}} \\
& =\check{B}^{\frac{1}{2}} \breve{A} \check{B}^{\frac{1}{2}}+I-\check{B}-B^{*} \widehat{B}^{\frac{1}{2}} \widehat{A} B \check{B}^{\frac{1}{2}} \\
& =\check{B}^{\frac{1}{2}} \check{A} \check{B}^{\frac{1}{2}}+B^{*} B \check{B}-B^{*} \widehat{B}^{\frac{1}{2}} \widehat{A} B \check{B}^{\frac{1}{2}} \\
& =\check{B}^{\frac{1}{2}} \check{A} \check{B}^{\frac{1}{2}}+B^{*} \widehat{B}^{\frac{1}{2}} B \check{B}^{\frac{1}{2}}-B^{*} \widehat{B}^{\frac{1}{2}} \widehat{A} B \check{B}^{\frac{1}{2}} \\
& =\check{B}^{\frac{1}{2}} \check{A} \check{B}^{\frac{1}{2}}+B^{*} \widehat{B}^{\frac{1}{2}}(I-\widehat{A}) B \check{B}^{\frac{1}{2}} \\
& =\check{B}^{\frac{1}{2}} \check{A} \check{B}^{\frac{1}{2}}+B^{*} \widehat{B}^{\frac{1}{2}} A A^{*} \widehat{A} B \check{B}^{\frac{1}{2}} \text {. }
\end{aligned}
$$

Thus $\check{B}^{\frac{1}{2}} A^{*} A \check{A} \check{B}^{\frac{1}{2}}+B^{*} \widehat{B}^{\frac{1}{2}} \widehat{A} B \check{B}^{\frac{1}{2}}=I-\check{B}^{\frac{1}{2}} \check{A} \check{B}^{\frac{1}{2}}-B^{*} \widehat{B}^{\frac{1}{2}} A A^{*} \widehat{A} B \check{B}^{\frac{1}{2}}$.

This shows that $I-\tilde{\Gamma}(B, A) \geq 0$. As $\|I-\tilde{\Gamma}(B, A)\|=\|P(I-Q)\|^{2} \leq 1$, it follows that $I-\tilde{\Gamma}(B, A) \leq I$ and hence $\tilde{\Gamma}(B, A) \geq 0$.

Now

$$
\begin{aligned}
\|P(I-Q)\|^{2} & =\|I-\tilde{\Gamma}(B, A)\| \\
& =\sup \left\{\|x\|-\|\tilde{\Gamma}(B, A) x\|: x \in H_{1},\|x\|=1\right\} \\
& =1-\inf \left\{\|\tilde{\Gamma}(B, A) x\|: x \in H_{1},\|x\|=1\right\} \\
& =1-m(\tilde{\Gamma}(B, A)) \\
& =1-m(\Gamma(B, A))^{2} .
\end{aligned}
$$

With a similar computation we can show that $\|Q(I-P)\|^{2}=1-m(\Gamma(A, B))^{2}$. The result now follows from the definition of $\theta_{0}(A, B)$ and $\theta(A, B)$. 
Remark 3.4. The formula in Theorem 3.3 improves the formula given by Nakamoto [18, Theorem 4.1]. In fact for $A, B \in \mathcal{B}(H)$, Nakamoto proved that

$$
\theta(A, B)=\sqrt{1-\min \{m(\tilde{\Gamma}(A, B)), m(\tilde{\Gamma}(B, A))\}},
$$

where

$$
\tilde{\Gamma}(A, B)=\check{B}^{\frac{1}{2}}\left(I+B^{*} A\right) \check{A}\left(I+A^{*} B\right) \check{B}^{\frac{1}{2}} .
$$

By the repeated use of relations in Lemma 2.9 it can be shown that $\tilde{\Gamma}(A, B)$ of (3.1) and $\tilde{\Gamma}(A, B)$ considered in Theorem 3.3 are the same.

Theorem 3.5. Let $A, B \in \mathcal{C}\left(H_{1}, H_{2}\right)$ be densely defined. Then

$$
\tilde{\theta}(A, B)=\sqrt{2\left(1-\sqrt{1-\theta(A, B)^{2}}\right)} .
$$

Proof. Let $c=\min \{m(\Gamma(A, B)), m(\Gamma(B, A))\}$. Then, by Theorem 3.3. $m(\Gamma(A, B))$ $=\sqrt{1-\theta^{2}(A, B)}$. By Theorem 3.1, we have

$$
\tilde{\theta}^{2}(A, B)=2(1-c)=2\left(1-\sqrt{1-\theta^{2}(A, B)}\right) .
$$

Hence $\tilde{\theta}(A, B)=\sqrt{2\left(1-\sqrt{1-\theta^{2}(A, B)}\right)}$.

Corollary 3.6. Let $A \in \mathcal{C}\left(H_{1}, H_{2}\right)$ be densely defined. Then $\theta(A)=1$ and $\tilde{\theta}(A)=$ $\sqrt{2}$.

Proof. By Theorem 3.3. $\theta(A)=\sqrt{1-\min \left\{m(\Gamma(A, 0))^{2}, m(\Gamma(0, A))^{2}\right\}}$. Here $\Gamma(A, 0)$ $=\check{A}^{\frac{1}{2}}=\Gamma(0, A)$ is self-adjoint. Hence $m(\Gamma(A, 0))=\inf \left\{\frac{1}{\sqrt{1+\lambda^{2}}}: \lambda \in \sigma\left(A^{*} A\right)\right\}=0$. Here we have used the fact that $\sigma\left(A^{*} A\right)$ is an unbounded since $A^{*} A$ is unbounded self-adjoint operator. By Theorems 3.1 and 3.3, $\theta(A)=\sqrt{2}$ and $\theta(A)=1$.

Corollary 3.7. Let $A, B \in \mathcal{C}\left(H_{1}, H_{2}\right)$ be densely defined. Then

(1) $\tilde{\theta}(A, B)=\tilde{\theta}\left(A^{*}, B^{*}\right)$.

(2) if $A$ and $B$ are one-to-one, then $\tilde{\theta}(A, B)=\tilde{\theta}\left(A^{-1}, B^{-1}\right)$.

Proof. This follows from Theorem 3.5 and the corresponding properties of $\theta(\cdot, \cdot)$ (see [12, Theorems 2.18, 2.20, pages 204-205] for details).

Corollary 3.8. Let $A, B \in \mathcal{C}\left(H_{1}, H_{2}\right)$ be densely defined. Then

$$
\theta(A, B) \leq \tilde{\theta}(A, B) \leq \sqrt{2} \theta(A, B) .
$$

Proof. The inequality $\theta(A, B) \leq \tilde{\theta}(A, B)$ follows from the definitions of $\theta(A, B)$ and $\tilde{\theta}(A, B)$. To prove the other inequality, it is enough to show that $2 \theta^{2}(A, B)-$ $\tilde{\theta}^{2}(A, B) \geq 0$. As $0 \leq \theta(A, B) \leq 1$, it follows that $0 \leq 1-\theta^{2}(A, B) \leq 1$. Hence

$$
\begin{aligned}
2 \theta^{2}(A, B)-\tilde{\theta}^{2}(A, B) & =2 \theta^{2}(A, B)-2\left(1-\sqrt{1-\theta^{2}(A, B)}\right) \\
& =2 \theta^{2}(A, B)-2+2 \sqrt{1-\theta^{2}(A, B)} \\
& =2\left(\sqrt{1-\theta^{2}(A, B)}-\left(\sqrt{1-\theta^{2}(A, B)}\right)^{2}\right) \\
& =2\left(\sqrt{1-\theta\left(^{2} A, B\right)}\right)\left(1-\sqrt{1-\theta^{2}(A, B)}\right) \\
& \geq 0 .
\end{aligned}
$$

This shows that the second relation is true.

Corollary 3.9. (1) The set $\left(\mathcal{B}\left(H_{1}, H_{2}\right), \tilde{\theta}(\cdot, \cdot)\right)$ is open in $\left(\mathcal{C}\left(H_{1}, H_{2}\right), \tilde{\theta}(\cdot, \cdot)\right)$. 
(2) If $A \in \mathcal{C}\left(H_{1}, H_{2}\right)$ is invertible with $A^{-1} \in \mathcal{B}\left(H_{2}, H_{1}\right)$ and $B \in \mathcal{B}\left(H_{1}, H_{2}\right)$ such that $\tilde{\theta}(A, B)<\frac{1}{\sqrt{1+\left\|A^{-1}\right\|^{2}}}$, then $B$ is invertible and $B^{-1} \in$ $\mathcal{B}\left(H_{2}, H_{1}\right)$. In particular, if $H_{1}=H_{2}=H$, then $\left\{A \in \mathcal{C}(H): A^{-1} \in \mathcal{B}(H)\right\}$ is open in $\mathcal{C}(H)$ with respect to the spherical gap metric.

Proof. The proof (11) follows by Corollary 3.8 and [12, Theorem 2.13, page 203]. The proof of (2) follows by Corollary [3.8 and [12, Theorem 2.21, page 205].

Corollary 3.10. The set of all bounded self-adjoint operators is a dense subset of the set of all unbounded self-adjoint operators with respect to the spherical gap metric.

Proof. The proof follows from Corollary 3.8 and [2, Proposition 1.6].

Example 3.11. Let $H:=$ the real space $L^{2}[0, \pi]$ of real-valued functions, $H^{1}=$ $\left\{x \in H: x\right.$ is absolutely continuous and $\left.x^{\prime} \in H\right\}$ and $H^{2}:=\left\{x \in H^{1}: x^{\prime} \in H^{1}\right\}$. Let $L x=d x / d t$, where $D(L)=\left\{x \in H^{1}: x(0)=x(\pi)=0\right\}$. Then $L \in \mathcal{C}(H)$ and $\overline{D(L)}=H$. For $n \in \mathbb{N}$, let $\phi_{n}(t):=\sqrt{\frac{2}{\pi}} \sin (n t), t \in[0, \pi]$ and $\psi_{n}(t):=$ $\sqrt{\frac{2}{\pi}} \cos (n t), t \in[0, \pi]$. Then $\left\{\phi_{n}\right\}_{n \in \mathbb{N}}$ forms an orthonormal basis for $H$.

It can be shown that $L^{*}=-d / d t, D\left(L^{*}\right)=H^{1}$ and $L^{*} L=-d^{2} / d t^{2}, D\left(L^{*} L\right)=$ $\left\{x \in H^{2}: x(0)=x(\pi)=0\right\}$. For $x \in D\left(L^{*} L\right)$ and $y \in H$, we have $x=\sum_{n=1}^{\infty}\left\langle x, \phi_{n}\right\rangle \phi_{n}$. Hence

$$
\begin{aligned}
L x & =\sum_{n=1}^{\infty} n\left\langle x, \phi_{n}\right\rangle \psi_{n}, \\
L^{*} L x & =\sum_{n=1}^{\infty} n^{2}\left\langle x, \phi_{n}\right\rangle \phi_{n}, \\
\left(I+L^{*} L\right) x & =\sum_{n=1}^{\infty}\left(1+n^{2}\right)\left\langle x, \phi_{n}\right\rangle \phi_{n}, \\
\check{L} y & =\sum_{n=1}^{\infty} \frac{1}{1+n^{2}}\left\langle y, \phi_{n}\right\rangle \phi_{n}, \\
\check{L} \frac{1}{2} y & =\sum_{n=1}^{\infty} \frac{1}{\sqrt{1+n^{2}}}\left\langle y, \phi_{n}\right\rangle \phi_{n} .
\end{aligned}
$$

We have $m(\Gamma(L, 0))=m\left(\check{L}^{\frac{1}{2}}\right)=\inf \left\{\frac{1}{\sqrt{1+n^{2}}}: n \in \mathbb{N}\right\}=0$. Hence by Theorems 3.1 and 3.3 , $\tilde{\theta}(L)=\sqrt{2}$ and $\theta(L)=1$.

Example 3.12. Let $H=\ell^{2}$. Let $D(A)=D(B)=\left\{\left(x_{j}\right) \in H: \sum_{j=1}^{\infty} j^{2}\left|x_{j}\right|^{2}<\infty\right\}$. Define $A: D(A) \rightarrow H$ by $A\left(\left(x_{j}\right)\right)=\left(j x_{j}\right)$, for all $\left(x_{j}\right) \in D(A)$ and

$$
B\left(\left(x_{j}\right)\right)=\left\{\begin{array}{rc}
-j x_{j} & \text { if } j=2 \\
j x_{j} & \text { if } j \neq 2 .
\end{array}\right.
$$


It can be shown that $A$ and $B$ are self-adjoint. For $\left(y_{j}\right) \in H$, we have

$$
\begin{aligned}
\check{A}\left(y_{j}\right) & =\widehat{A}\left(y_{j}\right)=\frac{1}{1+j^{2}} y_{j}=\check{B}\left(y_{j}\right)=\widehat{B}\left(y_{j}\right), \\
\check{A}^{\frac{1}{2}}\left(y_{j}\right) & =\frac{1}{\sqrt{1+j^{2}}} y_{j}=\check{B}^{\frac{1}{2}}\left(y_{j}\right), \\
A \check{A}\left(y_{j}\right) & =\left(\frac{j}{1+j^{2}} y_{j}\right), \\
A \check{A}^{\frac{1}{2}}\left(y_{j}\right) & =\left(\frac{j}{\sqrt{1+j^{2}}} y_{j}\right), \\
B \check{B}\left(y_{j}\right) & = \begin{cases}\frac{-j}{1+j^{2}} & \text { if } j=2, \\
\frac{j}{1+j^{2}} & \text { if } j \neq 2,\end{cases} \\
B \check{B}^{\frac{1}{2}}\left(y_{j}\right) & = \begin{cases}\frac{-j}{\sqrt{1+j^{2}}} & \text { if } j=2, \\
\frac{j}{\sqrt{1+j^{2}}} & \text { if } j \neq 2,\end{cases} \\
\Gamma(A, B)\left(y_{j}\right)=\left\{\begin{aligned}
-\frac{3}{5} y_{j} \quad \text { if } j=2, \\
y_{j} \quad \text { if } j \neq 2 .
\end{aligned}\right. &
\end{aligned}
$$

We have $m(\Gamma(A, B))=\inf \{|\lambda|: \lambda \in \sigma(\Gamma(A, B))\}=\frac{3}{5}=m(\Gamma(B, A))$. Hence by Theorems 3.3 and 3.1, we have $\theta(A, B)=\frac{4}{5}$ and $\tilde{\theta}(A, B)=\frac{2}{\sqrt{5}}$.

\section{REFERENCES}

1. N. I. Akhiezer and I. M. Glazman, Theory of linear operators in Hilbert space, Dover Publications. Inc., New York, 1993. Translated from the Russian and with a preface by Merlynd Nestell. Reprint of the 1961 and 1963 translations, two volumes bound as one. MR 1255973 (94i:47001)

2. Bernhelm Booss-Bavnbek, Matthias Lesch, and John Phillips, Unbounded Fredholm operators and spectral flow, Canad. J. Math. 57 (2005), no. 2, 225-250. MR 2124916 (2006a:58029)

3. H. O. Cordes and J. P. Labrousse, The invariance of the index in the metric space of closed operators, J. Math. Mech. 12 (1963), 693-719. MR0162142 (28:5341)

4. Dragana Cvetković, On gaps between bounded operators, Publ. Inst. Math. (Beograd) (N.S.) 72(86) (2002), 49-54. MR1997610 (2004d:47003)

5. Javad Faghih Habibi, The gap of the graph of a matrix, Linear Algebra Appl. 186 (1993), 55-57. MR 1217198 (94c:15039)

6. The spherical gap of the graph of a linear transformation, Proceedings of the 3rd ILAS Conference (Pensacola, FL, 1993), vol. 212/213, 1994, pp. 501-503. MR.1306995

7. Israel Gohberg, Peter Lancaster, and Leiba Rodman, Invariant subspaces of matrices with applications, Classics in Applied Mathematics, vol. 51, Society for Industrial and Applied Mathematics (SIAM), Philadelphia, PA, 2006, reprint of the 1986 original. MR2228089 (2007k:15001)

8. Simone Gramsch and Eberhard Schock, Ill-posed equations with transformed argument, Abstr. Appl. Anal. (2003), no. 13, 785-791. MR1996924 (2004f:47019)

9. C. W. Groetsch, Spectral methods for linear inverse problems with unbounded operators, J. Approx. Theory 70 (1992), no. 1, 16-28. MR.1168372 (93g:47011)

10. Inclusions for the Moore-Penrose inverse with applications to computational methods, Contributions in numerical mathematics, World Sci. Ser. Appl. Anal., vol. 2, World Sci. Publ., River Edge, NJ, 1993, pp. 203-211. MR1299760 (95h:65041)

11. Roger A. Horn, Chi-Kwong Li, and Dennis I. Merino, Distances between the graphs of matrices, Linear Algebra Appl. 240 (1996), 65-77. MR.1387286 (97e:15004)

12. Tosio Kato, Perturbation theory for linear operators, second ed., Springer-Verlag, Berlin, 1976, Grundlehren der Mathematischen Wissenschaften, Band 132. MR 0407617 (53:11389) 
13. William E. Kaufman, A stronger metric for closed operators in Hilbert space, Proc. Amer. Math. Soc. 90 (1984), no. 1, 83-87. MR722420 (85a:47010)

14. Fuad Kittaneh, On some equivalent metrics for bounded operators on Hilbert space, Proc. Amer. Math. Soc. 110 (1990), no. 3, 789-798. MR1027097 (91b:47017)

15. S. H. Kulkarni and G. Ramesh, A formula for gap between two closed operators, Linear Algebra and its Applications 432 (2010), 3012-3017.

16. S. H. Kulkarni, M. T. Nair and G. Ramesh, Some properties of unbounded operators with closed range, Proc. Indian Acad. Sci. Math. Sci. 118 (2008), no. 4, 613-625. MR2511129 (2010e:47006)

17. A. MacIntosh, Heinz inequalities and perturbation of spectral families, Macquarie math report (1979).

18. Ritsuo Nakamoto, Gap formulas of operators and their applications, Math. Japon. 42 (1995), no. 2, 219-232. MR.1356379 (96k:47033)

19. - The spherical gap of operators, Linear Algebra Appl. 251 (1997), 89-95. MR.1421267 (98e:47034)

20. Gert K. Pedersen, Analysis now, Graduate Texts in Mathematics, vol. 118, Springer-Verlag, New York, 1989. MR971256 (90f:46001)

21. Michael Reed and Barry Simon, Methods of modern mathematical physics. I, Functional Analysis, second ed., Academic Press. Inc. [Harcourt Brace Jovanovich Publishers], New York, 1980. MR 751959 (85e:46002)

22. Frigyes Riesz and Béla Sz.-Nagy, Functional analysis, Frederick Ungar Publishing Co., New York, 1955. MR0071727(17:175i)

23. Walter Rudin, Functional analysis, second ed., International Series in Pure and Applied Mathematics, McGraw-Hill Inc., New York, 1991. MR.1157815 (92k:46001)

Statistics and Mathematics Unit, Indian Statistical Institute Bangalore, Bangalore, INDIA 560059

E-mail address: ramesh@isibang.ac.in 